\title{
High Field Alloy, Thermoelectric, and mm Wave Studies of the Field Induced Superconducting State in $\lambda$-(BETS) $)_{2} \mathbf{F e}_{x} \mathbf{G a}_{1-x} \mathbf{C l}_{4}$
}

\author{
J.S. Brooks ${ }^{1}$, S. Uji ${ }^{2}$, E.S. Choi ${ }^{1}$, I.B. Rutel ${ }^{1}$, H. Kobayashi ${ }^{3,6}$, \\ A. Kobayashi ${ }^{4}, \mathrm{H}$. Tanaka ${ }^{5}$, and M. Tokumoto ${ }^{5,6}$ \\ ${ }^{1}$ National High Magnetic Field Laboratory and Physics Department, Florida State University, Tallahassee, FL 32310 USA \\ ${ }^{2}$ National Institute for Materials Science, Tsukuba, Ibaraki 305-0003, Japan \\ ${ }^{3}$ Institute for Molecular Science, Okazaki, 444-8585 Japan \\ ${ }^{4}$ Research Center for Spectrochemistry, The University of Tokyo, Bunkyo-ku, Tokyo 113-0033, Japan \\ ${ }^{5} 5$ Nanotechnology Research Institute, AIST, Tsukuba 305-8568, Japan \\ ${ }^{6}$ JST-CREST, Kawaguchi, Japan
}

Received on 23 May, 2003.

\begin{abstract}
Alloy studies in the $\pi$ - d organic conductor $\lambda$-(BETS) ${ }_{2} \mathrm{Fe}_{x} \mathrm{Ga}_{1-x} \mathrm{Cl}_{4}$ have given new insight into the nature of field induced superconductivity (FISC), since the mechanism of the FISC involves cancellation of the $\pi-$ $d$ exchange field by the external field. Alloying on the $\mathrm{Fe}_{x} \mathrm{Ga}_{1-x}$ site allows tuning of the exchange field, thereby influencing the FISC phase boundary. A brief review of the low temperature phases are given, and new high magnetic field thermoelectric and $\mathrm{mm}$ wave results that probe the low temperature ground state are presented.
\end{abstract}

The discovery of magnetic field induced superconductivity in $\lambda$-(BETS $)_{2} \mathrm{FeCl}_{4} \quad[1] \quad(\mathrm{BETS}=$ bis(ethylenedithio)tetraselenafulvalene) has drawn attention to the $\pi-d$ electron spin exchange mechanisms[2] in molecular systems where magnetic order in the d electron system strongly influences the behavior of the conducting $\pi$ electron system. The magnetic field dependent phase diagram of the $\lambda$-(BETS $)_{2} \mathrm{FeCl}_{4}$ material is shown in Fig. 1. For $\mathrm{H}=0$, and below the metal-insulator transition temperature $\left(T_{M I}=8.3 \mathrm{~K}\right), \lambda$-(BETS $)_{2} \mathrm{FeCl}_{4}$ enters a highly insulating antiferromagnetic (AFI) phase[3]. Below $T_{M I}$, a spin-flop transition to a canted antiferromagnetic (CAF) phase occurs near $1 \mathrm{~T}$, and above $11 \mathrm{~T}$, a paramagnetic metallic (PM) phase appears.

At higher magnetic fields, field induced superconductivity (FISC) is stabilized in $\lambda$-(BETS) ${ }_{2} \mathrm{FeCl}_{4}$ below $5 \mathrm{~K}$ between 18 and $45 \mathrm{~T}[1,4]$. The FISC state involves the cancellation of the exchange field $B_{J}$ by the external magnetic field $B_{\text {ext }}$, i.e. the effective internal field in the conduction electron layer is $B_{e f f}=B_{e x t}-\left|B_{J}\right|$ (i.e. the JaccarinoPeter or J-P effect[5,6], where for the $\pi-d$ discussed here, $B_{J}$ is negative.). Although the AFI-PM transition is nearly independent of field direction, the PM-FISC transition requires careful alignment of the field in the $a-c$ molecular planes to avoid orbital dissipation in the superconducting phase. Recently a similar FISC state has been reported in $\kappa$-(BETS $)_{2} \mathrm{FeBr}_{4}$ by Tanatar and co-workers.[11]
The more general $\lambda$-(BETS) ${ }_{2} \mathrm{Fe}_{x} \mathrm{Ga}_{1-x} \mathrm{Br}_{y} \mathrm{Cl}_{4-y}$ class of organic conductors, with localized magnetic moments at the anion sites, and conduction electrons in the molecularcation layers, exhibit competition between magnetic, metallic, insulating, and superconducting ground states. This is exemplified in Fig. $2 \mathrm{a}$ and $2 \mathrm{~b}$ where we show the zero field ground state phases for the $\lambda$-(BETS $)_{2} \mathrm{Fe}_{x} \mathrm{Ga}_{1-x} \mathrm{Cl}_{4}$ [8] and $\lambda$-(BETS $)_{2} \mathrm{FeBr}_{y} \mathrm{Cl}_{4-y}$ [9] series, respectively.

When the FISC was first discovered, it was tempting to anticipate that in order to exist at high fields, the superconducting state might arise from unconventional mechanisms such as triplet pairing. (See Lebed and Yamaji [10] for a recent theoretical treatment of possible low-D, high field, ground states.) However, the subsequent discovery that the superconducting phase was re-entrant at high magnetic fields, and that it had a "symmetry" around $32 \mathrm{~T}$ at the center of the FISC state, suggested that the Jaccarino-Peter mechanism should be seriously considered. Here, in simple terms, an external field of $32 \mathrm{~T}$ would cancel the corresponding exchange field $\mathbf{J}$ of the same order. Moreover, the superconducting phase diagram of the $\lambda$-(BETS $)_{2} \mathrm{GaCl}_{4}$ system (in-plane upper critical field vs. T), with no d-electron exchange component, looked strikingly similar to the upper half of the FISC phase between $32 \mathrm{~T}$ and $42 \mathrm{~T}$. This is illustrated in Fig. 1, where the upper critical field of $\lambda$ (BETS $)_{2} \mathrm{GaCl}_{4}$ from Tanatar et al. [7] has been transposed by $32 \mathrm{~T}$. Evidence that $B_{J}$ was indeed of order $32 \mathrm{~T}$ came 


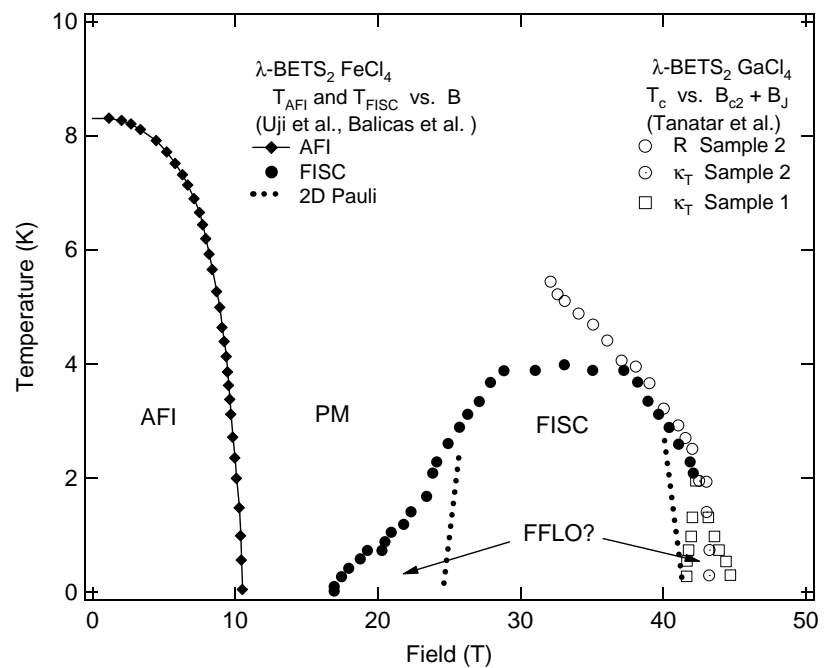

Figure 1. Magnetic field-dependent ground states of $\lambda$ (BETS $)_{2} \mathrm{FeCl}_{4}$ for $\mathrm{B} / / \mathrm{c}$-axis. The three main phases identified are the antiferromagnetic insulating (AFI), parmagnetic metal (PM), and the field induced superconducting (FISC) state. A spin-flop transition (not shown) occurs at about 1.2 T.[3] Solid symbols are determined from magnetoresitance data on a number of different samples from Refs. [1, 13, 4]. The dashed lines represent the expected FISC-PM phase boundary based on a standard 2D Paulilimited upper critical field. Open symbols represent the upper critical field of $\lambda$-(BETS $)_{2} \mathrm{GaCl}_{4}$ from resistance and thermal conductivity studies on two samples from Ref. [11]. Note that, for comparison of the two different systems, the upper critical field of the $\lambda$-(BETS $)_{2} \mathrm{GaCl}_{4}$ has been shifted by the $\lambda$-(BETS) ${ }_{2} \mathrm{FeCl}_{4}$ exchange field $B_{J}=32 \mathrm{~T}$. See text for discussion.

from Shubnikov-de Haas $(\mathrm{SdH})$ frequency $F$, measured for $\mathrm{B} / / \mathrm{b}$-axis[12]. From band structure calculations, there should be only one SdH frequency, characteristic of a single closed orbit. However, $F$ showed a Fe-concentration dependent splitting. From a simple spin-spitting argument, $\Delta F=g m_{e f f} / 4 m_{0} B_{J}$, for $\mathrm{x}=1$, where $\Delta F=128 \mathrm{~T}$, $m_{\text {eff }} / m_{0}=4$, and $\mathrm{g}=2$, the exchange field is $B_{J}=32$ T. Remarkably, $B_{J}$ depended linearly on the Fe concentration, and extrapolated to zero for $\mathrm{x}=0$. The connection between the $\lambda$-(BETS $)_{2} \mathrm{GaCl}_{4}$ and the $\lambda$-(BETS $)_{2} \mathrm{FeCl}_{4}$, and how the exchange field could be "tuned" from zero to its maximum value was conclusively demonstrated in a systematic investigation[13] of $\lambda$-(BETS $)_{2} \mathrm{Fe}_{x} \mathrm{Ga}_{1-x} \mathrm{Cl}_{4}$, the results of which are shown in Fig. 3. Here, for decreasing Fe concentration, the FISC state evolves smoothly into the $\lambda$-(BETS $)_{2} \mathrm{GaCl}_{4}$ superconducting phase. As shown in the figure, a phenomenological model by Fischer et al. [6] gives a good fit to the results. Unlike the case for the Chevrel phases, the AFI phase replaces the $\mathrm{B}=0$ superconducting phase. However, a recent investigation at low pressures (1 kbar or less) shows a small superconducting pocket near zero field $[14,15]$ very similar to that depicted in Fig. 3. Hence small pressures may stabilize the "hidden S phase".

Another remarkable aspect of the concentration dependence in Fig. 3 is the merging of the AFI, PM, and FISC phases near $\mathrm{x}=0.47$ for $\mathrm{T} \longrightarrow 0$. This suggests the pos- sibility of a quantum "tri-critical" point in the event that the transitions were found to be second order, for fine tuning of $\mathrm{x}$ (and maybe also pressure) in this region. For larger values of $\mathrm{x}$, there is no PM intervening state, and the AFI and FISC phases are connected along a single phase boundary.

The relatively simple J-P picture given above for the mechanism of field induced superconductivity in the $\lambda$-(BETS) $)_{2} \mathrm{Fe}_{x} \mathrm{Ga}_{1-x} \mathrm{Cl}_{4}$ system is only one part of a more complex low temperature ground state. For $\lambda$ (BETS $)_{2} \mathrm{FeCl}_{4}$, the metal insulating transition $T_{M I}$ involves an apparently coincident antiferromagnetic ordering and a Peierls transition, which, due to the correlated nature of the $\pi-d$ system, gives rise to a highly insulating phase where the resistivity increases by 6 or more orders of magnitude at low temperatures. It was discovered[9] that by alloying on the $C l_{1-y} B r_{y}$ site, these two transitions separate above $y=0.3$, as indicated in Fig. 2 above. Here the the AFI transition, detected by susceptibility, appears below the resistive metal-insulating transition. Work is presently underway to examine the effects of finite $y$ on the FISC state for $\lambda$-(BETS $)_{2} \mathrm{FeBr}_{y} \mathrm{Cl}_{4-y}$ where the Peierls and AF transitions are separated.[20]
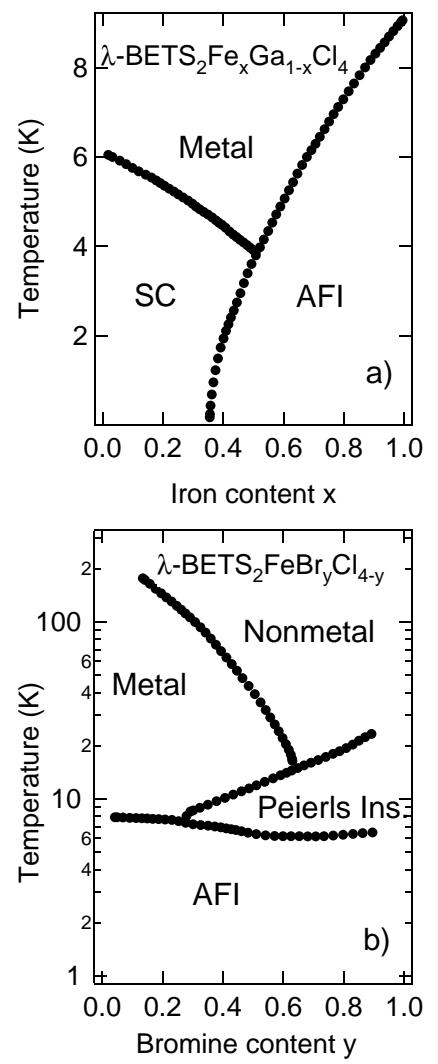

Figure 2. a) Zero field phase diagram of $\lambda$ (BETS) ${ }_{2} \mathrm{Fe}_{x} \mathrm{Ga}_{1-x} \mathrm{Cl}_{4}$ (after Ref.[8]) b) Zero field phase diagram of $\lambda$-(BETS) ${ }_{2} \mathrm{FeBr}_{y} \mathrm{Cl}_{4-y}$ (after Ref.[9]). Note that for $\mathrm{y}=1$, the $\lambda$ phase is not a stable compound. 


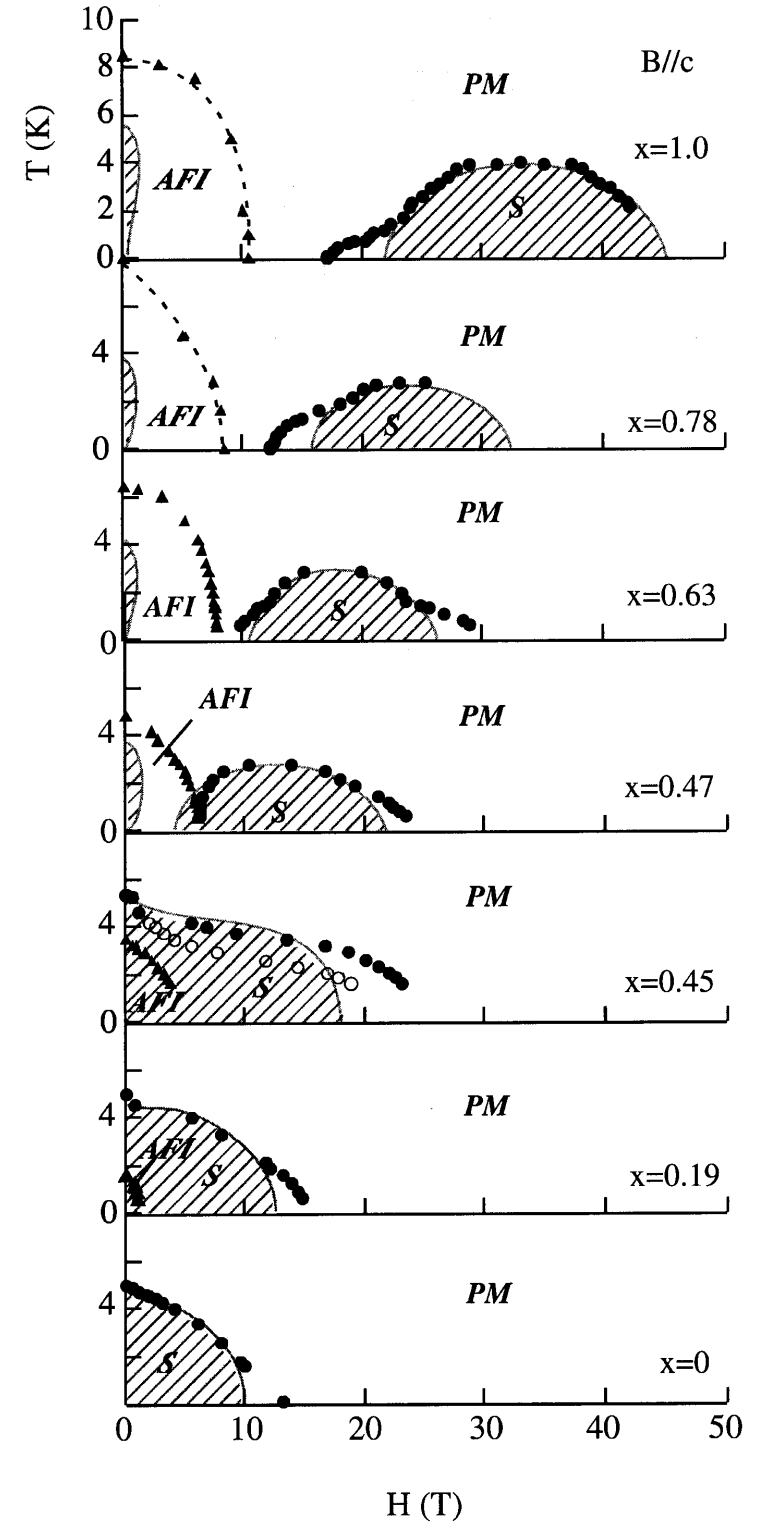

Figure 3. Evolution of AFI, PM, and FISC (S) ground states in $\lambda$ (BETS) ${ }_{2} \mathrm{Fe}_{x} \mathrm{Ga}_{1-x} \mathrm{Cl}_{4}$ for decreasing Fe concentration $x$. Shaded areas are theoretical superconducting phases predicted from the J-P model. See Ref. [13] for detailed discussion.

For magnetic fields aligned exactly in the conducting plane of a low-D superconductor, a Fulde-Ferrell-LarkinOvchinnikov (FFLO) ground state has been predicted.[16, 17] Under field alignment, there is no orbital, but only Zeeman effects, which will split the conduction band. In 1-D, in high enough magnetic fields, it is rigorous that singlet pairing with finite q-values gives a FFLO ground state, which is lower in energy than either the BCS or the normal state which are allowed for finite $2 \mathrm{D}$ dispersion. Hence the prediction is that the FFLO will appear for $\mathrm{T} \rightarrow 0$ between the regular BCS phase at lower fields and the normal state at higher fields. This result has been extended to $1-\mathrm{D}+$ small 2-D dispersion[18], but as the dimensionality increases, the FFLO will be suppressed. The FFLO is termed "inhomogeneous" since the order parameter will oscillate from max- imum to zero with a period of the order of the coherence length. Hence impurities and mean free path effects will influence the FFLO state. There is some indication that $\lambda$ (BETS) ${ }_{2} \mathrm{GaCl}_{4}$ may exhibit the FFLO state, based on recent thermal conductivity measurements [11] which show bifurcation of the upper critical field for $\mathrm{T} \rightarrow 0$. Moreover, the FISC state in $\lambda$-(BETS $)_{2} \mathrm{FeCl}_{4}$ shows, based on transport measurements, "wings" for $\mathrm{T} \rightarrow 0$ where $\pm B_{\text {eff }}$ approaches the two upper critical field lines. These features (wings) also appear in the alloy data in Fig. 3. This has motivated recent theoretical work which has considered the FFLO scenario for $\lambda$-(BETS $)_{2} \mathrm{FeCl}_{4}$, where the theory has been extended to include the effective field by Houzet et al.[21] In addition, Shimahara [22] has extended the treatment to allow both singlet and triplet pairing terms, which may be relevant in the case of $\lambda$-(BETS $)_{2} \mathrm{FeCl}_{4}$ since there is a profound proximity of magnetism. It is noteworthy that from the Dingle analysis, the mean free path in $\lambda$-(BETS) ${ }_{2} \mathrm{FeCl}_{4}$ can be as large as $100 \mathrm{~nm}$, or about an order of magnitude larger than the coherence length in $\lambda$-(BETS) ${ }_{2} \mathrm{GaCl}_{4}$ (of order $10 \mathrm{~nm}$ ), and hence the system is in the clean limit.[13] Although the comparison between experiment and theory is compelling, further work is needed to demonstrate the FFLO state convincingly in these materials. This is in part due to the quasitwo dimensional nature of the system, which complicates the more robust theoretical result obtained from a quasi-one dimensional approach to the FFLO ground state. (See also Ref. [13] for more detailed discussion.)

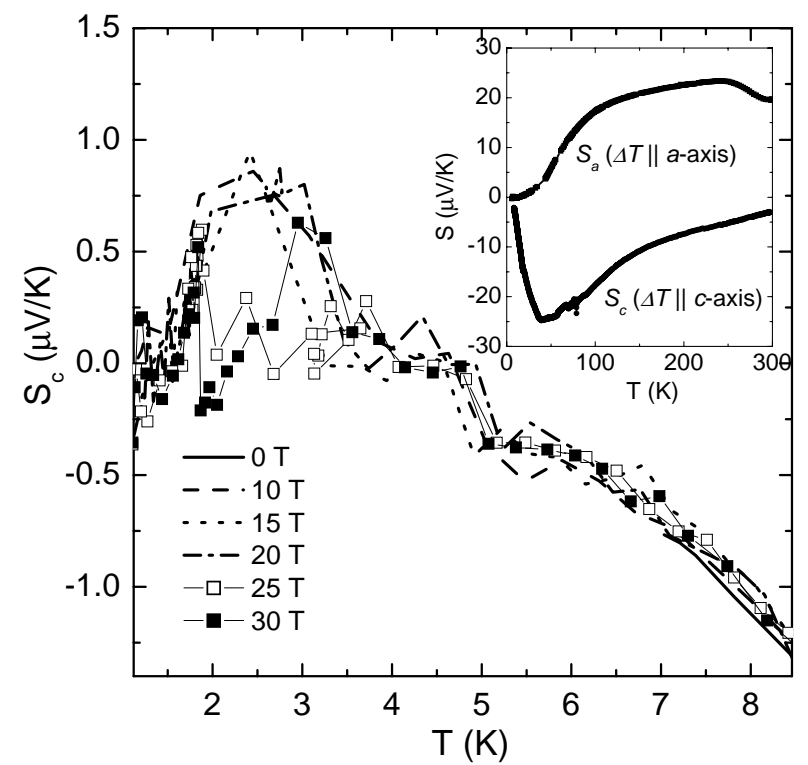

Figure 4. Magnetothermopower vs. temperature for $\lambda$ (BETS $)_{2} \mathrm{FeCl}_{4}$ for different fields below and above the FISC. The field direction and $\Delta T$ are along the c-axis. Inset: zero field thermopower for two crystallographic directions.

The $\lambda$-(BETS) ${ }_{2} \mathrm{Fe}_{x} \mathrm{Ga}_{1-x} \mathrm{Cl}_{4}$ FISC state described in Figs. 1 and 3 above have been mapped almost entirely with resistance measurements. (This is with the exception of torque measurements which show that there is a change in magnetization at the PM-FISC boundary.[1]) To 
further explore the nature of the FISC state, we have carried out magneto-thermoelectric power[23, 24, 25] and also mm wave-type investigations at high fields. In Fig. 4 we show the temperature dependence of the c-axis thermopower for $\lambda$-(BETS $)_{2} \mathrm{FeCl}_{4}$ for different fields. For fields in the FISC regime, the thermopower approaches zero below the PM-FISC phase boundary. In addition, the zero field thermopower shown in the inset shows the anisotropy of the Fermi surface, where, for the a-axis, the thermal gradient is in the direction of the hole orbits, and for the c-axis, it is directed normal to the open-orbit electron bands. Fig. 5 the field dependence for different temperatures is given. Here the thermopower again goes to zero in the FISC phase. Since the metallic thermopower is also vanishing for $\mathrm{T} \rightarrow 0$, the PM-FISC transition becomes less pronounced with decreasing temperature. A phase diagram, based on the thermopower studies (for $\mathrm{x}=1 \rightarrow$ FISC and AFI and $\mathrm{x}=0.8 \rightarrow$ AFI) is shown in Fig. 7 below. The important result of this study is that, as expected for a superconducting transition, the thermopower goes to zero below $T_{c}$. Hence this provides, additional confirmation, beyond resistance measurements, that the FISC is indeed a superconducting ground state.

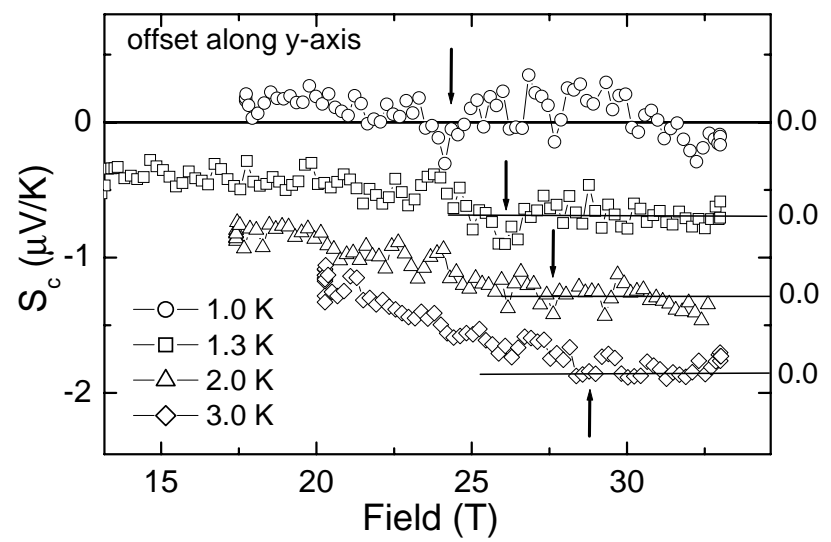

Figure 5. Magnetothermopower for $\lambda$-(BETS) ${ }_{2} \mathrm{FeCl}_{4}$ vs. field at different temperatures below the FISC transition. The field direction and $\Delta T$ are along the c-axis. Arrows indicate the PM-FISC boundary.

High field microwave studies have also been carried out vs. field and temperature up to $30 \mathrm{~T}$ to explore the nature of both the AFI and FISC states.[26] An example of the response of a perturbative cavity probe[27] at $66.76 \mathrm{GHz}$ is shown in Fig. 6. Here the c-axis of the sample is aligned with the field, but the $E_{a c}$ and $H_{a c}$ components of the electromagnetic resonant fields are mixed. At low fields, the $\mathrm{ESR}$,(and for $\mathrm{H} / /$ a-axis AFMR) resonances are observed. At higher fields, since the sample resistance decreases from an essentially insulating state to a metallic state as the field removes the AFI, the electromagnetic response passes from a depolarization to a skin-depth limited behavior, and the large dip is the so-called depolarization peak (DP). The temperature-field dependence of the depolarization behavior below AFI is shown in Fig. 7. Above the DP, but below the AFI-PM boundary, we observe additional structure in the signal, which we believe to arise from metastable states in the AFI phase. Finally, where the FISC state is stabilized, we find very little difference in the signal. Since the resistive transitions into the FISC are relatively broad, the change in the microwave signal may not be expected to be abrupt. However, systematic, higher field experiments are necessary to fully explore the $\mathrm{mm}$ wave behavior in the FISC state.

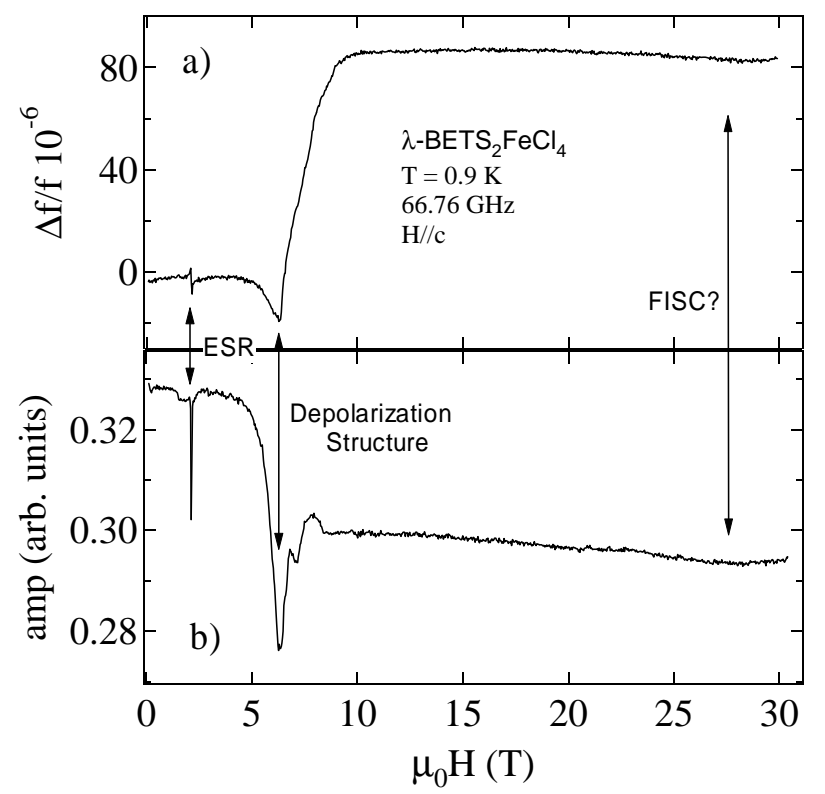

Figure 6. a) Frequency and b) amplitude response of $\mathrm{mm}$ wave cavity for $\lambda$-(BETS) $)_{2} \mathrm{FeCl}_{4}$ at $66.7 \mathrm{GHz}$ vs. field at $0.9 \mathrm{~K}$.

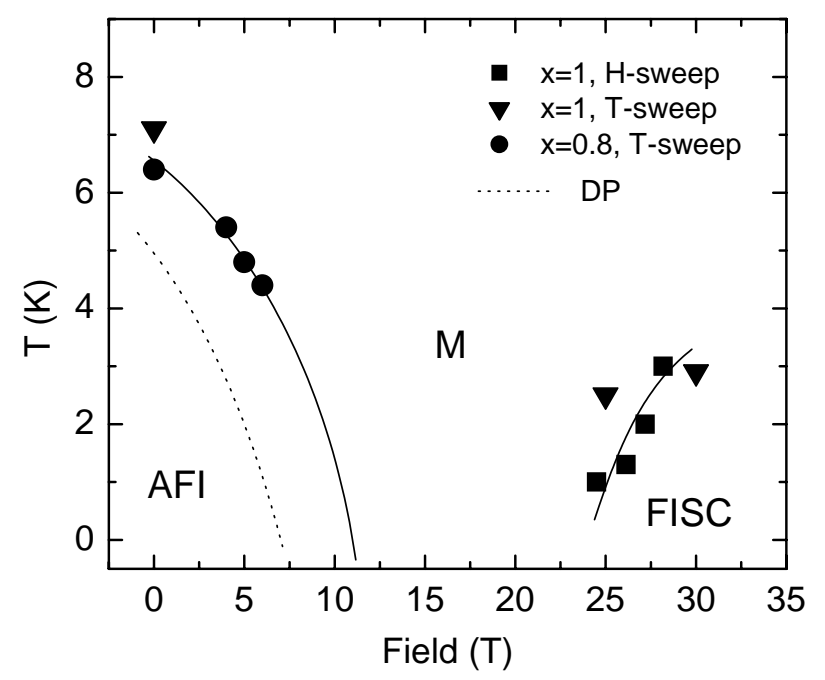

Figure 7. AFI and FISC phase boundaries determined from magnetothermopower measurements[25] (solid symbols) and behavior of the depolarization peak within the AFI from mm wave studies[26] (dashed line).

In summary, the $\lambda$-(BETS $)_{2} \mathrm{Fe}_{x} \mathrm{Ga}_{1-x} \mathrm{Br}_{y} \mathrm{Cl}_{4-y}$ class of organic-magnetic conductors allows a variety of novel ground states which can be modified with alloying, temperature, pressure, and magnetic field (including field direction). Moreover, the superconducting, Peierls, antiferromagnetic, polarized metallic, and field induced superconducting 
phases are non-trival due to the $\pi-d$ nature of the system. The anisotropic, low dimensional nature gives additional complexity, and novel high field superconducting ground states, such as FFLO and/or other unusual pairing mechanisms cannot be ruled out at this point. Even in spite of this, the connection between the pure $\lambda$-(BETS) ${ }_{2} \mathrm{FeCl}_{4}$ FISC phase and the $\lambda$-(BETS) ${ }_{2} \mathrm{GaCl}_{4} 2$-D superconductor phase, and how the $\pi-d$ exchange field can be tuned from the one extreme to the other, all in the context of the J-P model, is truly remarkable. There are still some very important experiments left to do in the FISC state, including specific heat, vortex dynamics, high field ESR on the $\pi$ electron system to check the behavior of $B_{\text {eff }}$ [28], and higher field transport above $42 \mathrm{~T}$ to firm up our understanding of the upper critical field behavior in the low temperature region.

\section{Acknowledgments}

We would like to thank K. Yang for helpful discussions. This work is supported by NSF-DMR 02-03532. The NHMFL is supported by a contractual agreement between the NSF and the State of Florida.

\section{References}

[1] S. Uji, H. Shinagawa, C. Terakura, T. Terashima, T. Yakabe, et al., Nature 410, 908 (2001).

[2] T. Mori and M. Katsuhara, J. Phys. Soc. Japan 71, 826 (2002).

[3] L. Brossard, R. Clerac, C. Coulon, M. Tokumoto, T. Ziman, D. K. Petrov, V. N. Laukhin, M. J. Naughton, A. Audouard, F. Goze, A. Kobayashi, H. Kobayashi, and P. Cassoux, Eur. Phys. J. B 1, 439 (1998).

[4] L. Balicas, J.S. Brooks, K. Storr, S. Uji, M. Tokumoto, H. Tanaka, H. Kobayashi, A. Kobayashi, V. Barzykin, L.P. Gor'kov, Phys. Rev. Lett. 87, 067002 (2001).

[5] V. Jaccarino and M. Peter, Phys. Rev. Lett. 9, 290 (1962).

[6] Ø. Fischer, H. W. Meul, M. G. Karkut, G. Remenyi, U. Welp and J. C. Piccoche, Phys. Rev. Lett. 55, 2972 (1985).

[7] M. A. Tanatar, M. Suzuki, T. Ishiguro, H. Tanaka, H. Fujiwara and H. Kobayashi, Physica C: Superconductivity, 388389, 613 (2003).

[8] H. Tanaka, H. Kobayashi, A. Kobayashi and P. Cassoux, Advanced Materials 12, 1685 (2000), and references therein.
[9] H. Akutsu, K. Kato, E. Ojima, H. Kobayashi, H. Tanaka, A. Kobayashi , P. Cassoux, Phys. Rev. B. 58, 9294 (1998).

[10] A.G. Lebed and K. Yamaji, Phys. Rev. Lett. 80, 2697 (1998).

[11] M. A. Tanatar, M. Suzuki, T. Ishiguro, H. Tanaka, H. Fujiwara and H. Kobayashi, T. Toito and J. Yamada, Synth. Met. 137, 1291 (2003).

[12] S. Uji, H. Shinagawa, C. Terakura, T. Terashima, T. Yakabe, Y. Terai, M. Tokumoto, A. Kobayashi, H. Tanaka and H. Kobayashi, Phys. Rev. B64, 024531/5 (2001).

[13] S. Uji, C. Terakura, T. Terashima, T. Yakabe, Y. Terai, Y. Imanaka, S. Yasuzuka, M. Tokumoto, F. Sakai, A. Kobayashi, H. Tanaka, H. Kobayashi, L. Balicas and J. S. Brooks, J. Phys. Soc. Japan 72, 369 (2003).

[14] J.S. Brooks, L. Balicas, K.A. Storr, H. Kobayashi, H. Tanaka, A. Kobayashi, S. Uji, and M. Tokumoto, Synth. Met. 133134, 485 (2003).

[15] L. Balicas, J.S. Brooks, K. Storr, S. Uji, M. Tokumoto, H. Tanaka, H. Kobayashi, A. Kobayashi, V. Barzykin, L.P. Gor'kov, Int. J. Mod. Phys. B16 (20-22), 3101 (2000).

[16] P. Fulde and R.A. Ferrell, Phys. Rev. 135 A550 (1964).

[17] A.I. Larkin and Y.N. Ovchinnikov, Sov. Phys. JETP 20, 762 (1965).

[18] K. Yang, Phys. Rev. B 63, 140511 (2001).

[19] E. Negishi, H. Uozaki, Y. Ishizaki, H. Tsuchiya, S. Endo, Y. Abe, H. Matsui, and N. Toyota, Synth. Met. 9439 (2002).

[20] S. Uji et al., to be published.

[21] M. Houzet, A. Buzdin, L. Bulaevskii and M. Maley, Phys. Rev. Lett. 88, 227001/4 (2002).

[22] H. Shimahara, J. Phys. Soc. Japan 71, 1644 (2002).

[23] E. S. Choi, J. S. Brooks, J. S. Qualls, et al., Rev. Sci. Instrum. 72, $2392(2001)$

[24] E. S. Choi, J. S. Brooks, and J. S. Qualls, Phys. Rev. B 65, 205119 (2002).

[25] E.S. Choi et al., to be published.

[26] I.Rutel, S. Okubo, J. Brooks, E. Jobiliong, H. Kobayashi, A. Kobayashi , and H. Tanaka, cond-mat/0207747 and to be published.

[27] S. Hill, N. S. Dalal and J. S. Brooks, Applied Magnetic Resonance 16, 237 (1999).

[28] O. Cepas, R. H. McKenzie and J. Merino,Phys. Rev. B 65, 100502(R) (2002). 